\title{
Opers of higher types, Quot-schemes and Frobenius instability loci
}

\author{
Kirti Joshi and Christian Pauly
}

\begin{abstract}
In this paper we continue our study of the Frobenius instability locus in the coarse moduli space of semi-stable vector bundles of rank $r$ and degree 0 over a smooth projective curve defined over an algebraically closed field of characteristic $p>0$. In a previous paper we identified the "maximal" Frobenius instability strata with opers (more precisely as opers of type 1 in the terminology of the present paper) and related them to certain Quot-schemes of Frobenius direct images of line bundles. The main aim of this paper is to describe for any integer $q \geq 1$ a conjectural generalization of this correspondence between opers of type $q$ and Quot-schemes of Frobenius direct images of vector bundles of rank $q$. We also give a conjectural formula for the dimension of the Frobenius instability locus.
\end{abstract}

Keywords. Vector bundles, Semi-stability, Frobenius map, Quot-scheme, Oper

2020 Mathematics Subject Classification. 14H60, 14D20, 14G17

\section{[Français]}

Opers de type supérieur, schémas Quot et lieux d'instabilité de Frobenius

Résumé. Nous continuons l'étude du lieu d'instabilité de Frobenius dans l'espace de modules grossier des fibrés semi-stables de rang $r$ et degré 0 sur une courbe projective lisse définie sur un corps algébriquement clos de caractéristique $p>0$. Dans un article précédent, nous avons identifié les strates « maximales » d'instabilité de Frobenius avec certains opers (de type 1, avec la terminologie du présent article) et avons montré leur lien avec certains schémas Quot d'images directes sous Frobenius de fibrés en droites. Le principal objectif de cet article est de décrire, pour tout $q \geq 1$, une généralisation conjecturale de cette correspondance entre opers de type $q$ et schémas Quot d'images directes sous Frobenius de fibrés de rang $q$. Nous donnons également une formule conjecturale pour la dimension du lieu d'instabilité de Frobenius.

Received by the Editors on August 28, 2019, and in final form on June 30, 2020.

Accepted on September 13, 2020.

\section{Kirti Joshi}

Mathematics Department, University of Arizona, 617 N Santa Rita, Tucson 85721-0089, USA

e-mail: kirti@math.arizona.edu

Christian Pauly

Laboratoire de Mathématiques J.A. Dieudonné, UMR 7351 CNRS, Université de Nice Sophia-Antipolis, 06108 Nice Cedex 02, France e-mail: pauly@unice.fr

KJ would like to thank the University of Nice Sophia-Antipolis for financial support of research visits in June 2013 and June 2016.

(c) by the author(s)

This work is licensed under http://creativecommons.org/licenses/by-sa/4.0/ 


\section{Contents}

1. Introduction. . . . . . . . . . . . . . . . . . . . . . . . . . . . . . . . 2

2. Preliminaries on Quot-schemes . . . . . . . . . . . . . . . . . . . . . . . . 3

3. A finiteness theorem . . . . . . . . . . . . . . . . . . . . . . . . . . . . 5

4. Opers of type $q$. . . . . . . . . . . . . . . . . . . . . . . . . . . . . . . . . . . . . . 7

5. Properties of the Quot-scheme $\operatorname{Quot}^{r, 0}\left(F_{*}(Q)\right)$ when $q=1$. . . . . . . . . . . . . . 11

6. The Quot-scheme $\operatorname{Quot}^{r, 0}\left(F_{*}(Q)\right)$ is bigger than expected when $q \geq 2$. . . . . . . . . 13

7. A characterization of dormant opers of type $q$. . . . . . . . . . . . . . . . . . . . . . 14

8. Dimension of Frobenius instability loci . . . . . . . . . . . . . . . . . . . . . 16

References. . . . . . . . . . . . . . . . . . . . . . . . . . . 17

\section{Introduction}

Let $p$ be a prime number. Let $k$ be an algebraically closed field of characteristic $p$. Let $X / k$ be a connected, smooth projective curve over $k$. We will write $F: X \rightarrow X$ for the absolute Frobenius morphism of $X$. A foundational classical problem in the theory of vector bundles on smooth, projective curves is the following:

Problem 1.1. Describe the Frobenius instability locus, i.e. the locus of all stable (and also semi-stable) vector bundles $V$ over $X$ such that $F^{*}(V)$ is not semi-stable.

This goal has been partially achieved by us in [JP15] where, for $p \gg 0$ we provided an explicit construction of all stable bundles $V$ such that $F^{*}(V)$ is not semi-stable. By a well-known theorem of [Sha76], one may equip the moduli space of semi-stable vector bundles of fixed degree and rank by a stratification defined using Harder-Narasimhan polygons of $F^{*}(V)$. The main problem addressed in the present paper is the finer problem:

Problem 1.2. Describe all Frobenius instability strata.

One of the important results of [JP15] asserts that there is an explicit polygon, called the oper polygon (which is always attained in every genus and rank) with the property that the Harder-Narasimhan polygon of every vector bundle $F^{*}(V)$, with $V$ stable, lies on or strictly below the oper polygon.

So to clarify the second problem, one can for example ask: do all possible Harder-Narasimhan polygons occur? This seems substantially more difficult and complete results are presently available only for $p=2$ (see [JX00]). We have obtained a number of results in small rank, genus, and characteristic, which we will report in a companion paper under preparation. Recently [Li19] has also studied the genus-2, rank-3, characteristic- 3 case in detail (our small genus, rank, characteristic results include his results, but by methods quite different from his).

The description which we provided in our answer to Problem 1.1 is that all Frobenius-destabilized stable bundles arise from suitable quotients of bundles of the form $F_{*}(Q)$ with $Q$ a stable vector bundle. In all the small genus, small rank, small characteristic situations, the vector bundle $Q$ is of $\operatorname{rank} 1$. If $\operatorname{rk}(Q)=1$, 
the Quot-schemes Quot ${ }^{r, 0}\left(F_{*}(Q)\right)$ parameterizing subsheaves of given rank $r$ and degree 0 of $F_{*}(Q)$ are substantially better behaved than for general rank : in fact, one of the main results of [JP15] says that if $\operatorname{rk}(Q)=1$ then the dimension of $\operatorname{Quot}^{r, 0}\left(F_{*}(Q)\right)$ is 0 if its expected dimension is 0 , and its $k$-rational points correspond to opers (of type 1). As shown in section 6 , this fails if $\operatorname{rk}(Q)>1$. The existence of these higher-dimensional components of Quot ${ }^{r, 0}\left(F_{*}(Q)\right)$ stems from certain line subbundles $L \hookrightarrow Q$ of sufficiently high degree, which induce natural embeddings $\operatorname{Quot}^{r, 0}\left(F_{*}(L)\right) \hookrightarrow \operatorname{Quot}^{r, 0}\left(F_{*}(Q)\right)$.

In the present paper we lay the ground work for addressing the second problem and its finer special cases. The key tool we introduce here is the notion of opers of type $q$ and rank $\ell q$ (see [JP15, Definition 3.1.1], recalled in Section 4). This notion generalizes the notion of an oper $\left(V, \nabla, V_{\bullet}\right)$ studied by [BD00] and consisting of a vector bundle $V$ equipped with an integrable connection $\nabla$ and a full flag $V_{\bullet}$ satisfying some transversality conditions. An oper of type $q$ should be thought of as the bundle analog of the parabolic induction (and its adjoint the Jacquet Functor) in the theory of automorphic forms - the parabolic in the present case is of type given by the $\ell$-tuple $(q, \ldots, q)$, which corresponds to a partial flag whose associated quotients are all of rank $q$. Given an oper of type $q$ and rank $\ell q$ we naturally obtain a rank- $q$ bundle $Q$ as first quotient of the oper filtration.

In the case $\operatorname{rk}(Q)>1$ we conjecture (Conjecture 7.6) a similar statement assuming that one restricts attention to "non-degenerate" subsheaves of $F_{*}(Q)$, i.e. excluding in particular the above-mentioned sub-Quotschemes Quot ${ }^{r, 0}\left(F_{*}(L)\right)$. More precisely, we conjecture that, if the expected dimension of $\operatorname{Quot}^{\ell q, 0}\left(F_{*}(Q)\right)$ is 0 , then dormant opers of type $q=\operatorname{rk}(Q)$ form a non-empty open subset of dimension 0 of the Quot-scheme Quot $^{\ell q, 0}\left(F_{*}(Q)\right)$, which has, as mentioned above, components of dimension $>0$. Here dormant means that the $p$-curvature of the connection $\nabla$ is zero. We check that this conjecture holds when $Q$ is a semi-stable direct sum of $q$ line bundles (Theorem 3.5).

Finally, somewhat independently of the previous considerations, we give a conjecture for the dimension of the Frobenius instability locus $\mathcal{J}(r)$ in the coarse moduli space of semi-stable rank $r$ and degree 0 vector bundles. This conjecture says that

$$
\operatorname{dim} \mathcal{J}(r) \geq\left(r^{2}-r+1\right)(g-1)-(r-1) .
$$

The conjecture holds for $r=2$. We also conjecture that a general vector bundle in $\mathcal{J}(r)$ has "minimal" Harder-Narasimhan filtration (Conjecture 8.3).

Acknowledgements. We would like to thank the referee for useful comments and suggestions.

\section{Preliminaries on Quot-schemes}

In what follows, the following notations and assumptions will be in force. Let $X$ be a smooth, projective curve of genus $g \geq 2$ over an algebraically closed field $k$ of characteristic $p>0$. Let $F: X \rightarrow X$ be the absolute Frobenius morphism of $X$. For a vector bundle $V$, we shall write $V^{*}$ for its dual $\mathscr{H} \circ \mathrm{om}\left(V, \mathcal{O}_{X}\right)$.

Suppose $\theta$ is a theta-characteristic for $X$. Let $\mathscr{G}_{2}$ be the unique non-split extension of $\theta^{-1}$ by $\theta$ : this bundle depends on the choice of the theta-characteristic $\theta$ but our notation suppresses this dependence. For $r<p$ let $\mathscr{G}_{r}=\operatorname{Sym}^{r-1}\left(\mathscr{G}_{2}\right)$ which will be called the Gunning bundle of rank $r$. This is an indecomposable bundle of degree zero and trivial determinant.

We recall two formulas from [JRXY06]. Let $Q$ be a vector bundle of rank $q$ and slope $\mu(Q)$ on $X$. Then

$$
\operatorname{deg}\left(F_{*}(Q)\right)=q \mu(Q)+q(p-1)(g-1)
$$

and equivalently

$$
\mu\left(F_{*}(Q)\right)=\frac{\mu(Q)}{p}+\frac{(p-1)(g-1)}{p} .
$$


The first is a consequence of the Riemann-Roch formula $\chi(V)=\operatorname{deg}(V)+\operatorname{rk}(V)(1-g)$ for a vector bundle $V$ of rank $\operatorname{rk}(V)$ and degree $\operatorname{deg}(V)$ on $X$ and the fact that $\chi(V)=\chi\left(F_{*}(V)\right)$ for the finite map $F$. The second is equivalent to the first.

Let $Q$ be a stable bundle on $X$ of rank $q$ and slope $\mu(Q)=\mu<0$. Let $r \geq 1$ be an integer. For a coherent sheaf $V$ and a surjection $V \rightarrow G$ to a coherent sheaf, we will say that $G$ has codegree $d$ if the kernel $\operatorname{ker}(V \rightarrow G)$ has degree $d$. Similarly we will say that $G$ has corank $r$ if $\operatorname{ker}(V \rightarrow G)$ has rank $r$. Let Quot ${ }^{r, 0}\left(F_{*}(Q)\right)$ be the Quot-scheme of quotients of $F_{*}(Q)$ of codegree 0 and corank $r$. If $V \rightarrow G$ is a quotient with kernel $E$ we will habitually write $[E]$ for the corresponding point of the relevant Quot-scheme corresponding to this quotient.

Let $[E] \in \operatorname{Quot}^{r, 0}\left(F_{*}(Q)\right)$ be a point of the Quot-scheme. Then we define the integer

$$
e(E)=\exp \cdot \operatorname{dim}_{[E]}\left(\operatorname{Quot}^{r, 0}\left(F_{*}(Q)\right)\right)=\chi\left(\mathscr{H} \circ \mathrm{om}\left(E, F_{*}(Q) / E\right)\right) .
$$

The integer $e(E)$ is called the expected dimension of $\operatorname{Quot}^{r, 0}\left(F_{*}(Q)\right)$ at the point $[E]$ (see [HL10, Chapter 2]).

Proposition 2.4. Let $r \geq 1$ be an integer. Let $Q$ be a vector bundle on $X$ of rank $q \leq r-1$ and slope $\mu(Q)=\mu$. Let $[E] \in \operatorname{Quot}^{r, 0}\left(F_{*}(Q)\right)$ be a point of the Quot-scheme. Then the following assertions hold.

(1) The expected dimension of $\operatorname{Quot}^{r, 0}\left(F_{*}(Q)\right)$ at $[E]$ is

$$
e(E)=r \operatorname{deg}(Q)+\left(r^{2}-q r\right)(g-1) .
$$

(2) We have $e(E)=0$ if and only if

$$
\operatorname{deg}(Q)=(r-q)(1-g)
$$

(3) In particular, if $r=\ell q$ for some integer $\ell \geq 2$, then $e(E)=0$ if and only if

$$
\operatorname{deg}(Q)=q(\ell-1)(1-g) .
$$

(4) Moreover if $\mu=-\frac{1}{q}$ (equivalently $\operatorname{deg}(Q)=-1$ ) then

$$
e(E)=\left(r^{2}-q r\right)(g-1)-r .
$$

(5) If $\operatorname{deg}(Q)=(r-q)(1-g)+d$ with $d \geq 0$, then

$$
e(E)=r d .
$$

Proof. It is enough to prove the first assertion as the rest are immediate consequences of the first. Let $[E] \in \operatorname{Quot}^{r, 0}\left(F_{*}(Q)\right)$ be a point corresponding to a quotient $F_{*}(Q) \rightarrow G$. Then by definition the expected dimension is given by $e(E)=\chi(\mathscr{H} \circ m(E, G))$. By the Riemann-Roch formula we have

$$
\chi(\mathscr{H} \circ \mathrm{om}(E, G))=\operatorname{deg}\left(E^{*} \otimes G\right)+\operatorname{rk}(E) \operatorname{rk}(G)(1-g)
$$

which gives

$$
e(E)=r \operatorname{deg}(G)+r(p q-r)(1-g) .
$$

As $E$ has degree zero, so $\operatorname{deg}(G)=\operatorname{deg}\left(F_{*}(Q)\right)=q \mu+q(p-1)(g-1)$. Substituting this in the above equation and simplifying the result gives the asserted formula.

Proposition 2.5. Let $Q$ be a semistable bundle of rank $q$ and $\operatorname{deg}(Q)=(r-q)(1-g)+d$ with $q<r<p q$ and $d \geq 0$. Then

(1) The Quot-scheme $\operatorname{Quot}^{r, 0}\left(F_{*}(Q)\right)$ is non-empty.

(2) Any irreducible component of $\mathrm{Quot}^{r, 0}\left(F_{*}(Q)\right)$ has dimension $\geq r d$.

(3) If $p>\max \left(2 r(r-1)(g-1), \frac{2 r d}{q}\right)$ then for any $[E] \in \operatorname{Quot}^{r, 0}\left(F_{*}(Q)\right)$, the quotient $F_{*}(Q) / E$ is torsion-free, i.e., $E \hookrightarrow F_{*}(Q)$ is a subbundle. 
Proof. The assertion (1) is [JP15, Proposition 2.3.2]. The assertion (2) is [JP15, Proposition 2.3.4]. So it remains to prove (3). Let $E^{\prime}$ denote the saturation of $E$ in $F_{*}(Q)$. Then $\mu\left(E^{\prime}\right) \geq 0$. Assume on the contrary that $E^{\prime} \neq E$, i.e., $\mu\left(E^{\prime}\right)>0$. Then we invoke [JP15, Proposition 4.2.1] with $n=r$ and $\delta=\frac{1}{2 r}$, and we note that $\mu(Q)=\frac{(r-q)(1-g)}{q}+\frac{d}{q}$ and that we have the following inequality

$$
\frac{\mu(Q)}{p}+\delta=\frac{(r-q)(1-g)}{p q}+\frac{d}{p q}+\frac{1}{2 r}<\frac{1}{2 r}+\frac{1}{2 r}=\frac{1}{r} .
$$

Then [JP15, Proposition 4.2.1] implies that

$$
0<\mu\left(E^{\prime}\right)<\frac{\mu(Q)}{p}+\delta<\frac{1}{r}
$$

which leads to a contradiction, $\operatorname{since} \operatorname{rk}\left(E^{\prime}\right)=\operatorname{rk}(E)=r$.

\section{A finiteness theorem}

We start with a lemma.

Lemma 3.1. Let $Q$ be a line bundle of degree $-(r-1)(g-1)$ with $r \geq 2$. If $r^{\prime}<r$ then

$$
\operatorname{Quot}^{r^{\prime}, 0}\left(F_{*}(Q)\right)=\emptyset \text {. }
$$

Proof. Suppose the assertion is not true. Then we have $\operatorname{Quot}^{r^{\prime}, 0}\left(F_{*}(Q)\right) \neq \emptyset$. Consider a subsheaf $E \in$ $\operatorname{Quot}^{r^{\prime}, 0}\left(F_{*}(Q)\right)$. We will use the notation of $\left[\mathrm{PP} 15\right.$, Lemma 3.4.2]. Let $W=F^{*}(E)$ and equip it with the filtration induced by the natural filtration $V_{\bullet}$ on the bundle $V=F^{*}\left(F_{*}(Q)\right)$. We shall denote the latter by $0=W_{m+1} \subset W_{m} \subseteq \cdots \subseteq W_{1} \subseteq W_{0}=W$. Let $r_{i}=\operatorname{rk}\left(W_{i} / W_{i+1}\right)$. Then $1=r_{0} \geq r_{1} \geq \cdots \geq r_{m} \geq 1$. So $r_{i}=1$ for all $i \geq 0$. Now we have the following inequalities

$$
\begin{aligned}
0=\operatorname{deg}(W)=\sum_{i=0}^{m} \operatorname{deg}\left(W_{i} / W_{i+1}\right) & \leq \sum_{i=0}^{m} \operatorname{deg}\left(V_{i} / V_{i+1}\right), \\
0 & \leq \sum_{i=0}^{m}(\operatorname{deg}(Q)+i(2 g-2)), \\
0 & \leq(m+1) \operatorname{deg}(Q)+\frac{m(m+1)}{2}(2 g-2) .
\end{aligned}
$$

As $\operatorname{rk}(E)=r^{\prime}$ so $m=r^{\prime}-1$ and hence the last inequality can be written as

$$
0 \leq r^{\prime}(r-1)(1-g)+r^{\prime}\left(r^{\prime}-1\right)(g-1)=r^{\prime}(g-1)\left(r^{\prime}-1-r+1\right)=r^{\prime}\left(r^{\prime}-r\right)(g-1)<0 .
$$

Thus we have arrived at a contradiction.

We will now prove a finiteness theorem for the Quot-scheme Quot ${ }^{\ell \mathrm{q}, 0}\left(F_{*}(\mathrm{Q})\right)$ when $Q$ is a decomposable bundle. Assume that $q \geq 2, \ell \geq 2$ and that $p>r(r-1)(r-2)(g-1)$ for $r=\ell q$. Let $L_{1}, \ldots, L_{q}$ be $q$ distinct line bundles of degree $-(\ell-1)(g-1)$. We denote by $Q$ the decomposable bundle

$$
Q=\oplus_{i=1}^{q} L_{i} \text { and } Q_{j}=\oplus_{i \neq j} L_{i} \subset Q .
$$

Then for any $j=1, \ldots, q$ we have an inclusion

$$
\operatorname{Quot}^{\ell \mathrm{q}, 0}\left(F_{*}\left(\mathrm{Q}_{\mathrm{j}}\right)\right) \hookrightarrow \operatorname{Quot}^{\ell \mathrm{q}, 0}\left(F_{*}(\mathrm{Q})\right) \text {. }
$$


By Proposition 2.5(2) we have

$$
\operatorname{dim} \operatorname{Quot}^{\ell q, 0}\left(F_{*}\left(\mathrm{Q}_{\mathrm{j}}\right)\right) \geq q \ell(\ell-1)(g-1)>0 .
$$

We denote by $\Omega(Q)$ the residual component of these $q$ Quot-schemes in $\operatorname{Quot}^{\ell \mathrm{q}, 0}\left(F_{*}(\mathrm{Q})\right)$, i.e., we have a union

$$
\operatorname{Quot}^{\ell \mathrm{q}, 0}\left(F_{*}(\mathrm{Q})\right)=\bigcup_{j=1}^{q} \operatorname{Quot}^{\ell \mathrm{q}, 0}\left(F_{*}\left(\mathrm{Q}_{\mathrm{j}}\right)\right) \cup \Omega(Q) \text {. }
$$

Theorem 3.5. We consider the morphism

$$
\Phi: \prod_{i=1}^{q} \operatorname{Quot}^{\ell, 0}\left(F_{*}\left(\mathrm{~L}_{\mathrm{i}}\right)\right) \longrightarrow \operatorname{Quot}^{\ell \mathrm{q}, 0}\left(F_{*}(\mathrm{Q})\right)
$$

defined by

$$
\left(S_{i} \subset F_{*}\left(L_{i}\right)\right)_{i=1}^{q} \longmapsto\left(\oplus_{i=1}^{q} S_{i} \subset F_{*}(Q)\right) .
$$

Then $\Phi$ induces a bijection at the level of $k$-rational points

$$
\prod_{i=1}^{q} \operatorname{Quot}^{\ell, 0}\left(F_{*}\left(\mathrm{~L}_{\mathrm{i}}\right)\right)(k) \simeq \Omega(Q)(k) .
$$

In particular $\Omega(Q)(k) \neq \emptyset$ and

$$
\operatorname{dim} \Omega(Q)=0
$$

Proof. Let $[E] \in \operatorname{Quot}^{\ell \mathrm{q}, 0}\left(F_{*}(\mathrm{Q})\right)$. We will denote by $E_{i}=\pi_{i}(E)$ the image of $E$ under the projection $\pi_{i}: F_{*}(Q) \rightarrow F_{*}\left(L_{i}\right)$. First we note the equivalence $[E] \in \operatorname{Quot}^{\ell \mathrm{q}, 0}\left(F_{*}\left(\mathrm{Q}_{\mathrm{i}}\right)\right)$ if and only if $E_{i}=\pi_{i}(E)=0$. Therefore, for any $[E] \in\left(\operatorname{Quot}^{\ell \mathrm{q}, 0}\left(F_{*}(\mathrm{Q})\right) \backslash \bigcup_{i=1}^{q} \operatorname{Quot}^{\ell \mathrm{q}, 0}\left(F_{*}\left(\mathrm{Q}_{\mathrm{i}}\right)\right)\right)$ we have $E_{i} \neq 0$ for $i=1, \ldots, q$. Moreover, by [JP15, Proposition 4.5.1 and Theorem 4.1.1] the vector bundles $E$ and $E_{i}$ are semi-stable and their degrees are zero. We now consider the kernel

$$
K_{i}=\operatorname{ker}\left(E \rightarrow \oplus_{j \neq i} E_{j}\right) .
$$

Then clearly $K_{i} \subset F_{*}\left(L_{i}\right)$. Note that $K_{i}=E \cap F_{*}\left(L_{i}\right)$ and so $K_{i} \cap K_{j}=0$ for $i \neq j$. Moreover, by our assumption on $E$, we have $K_{i} \neq 0$ for all $i=1, \ldots, q$. As the bundles $E$ and $E_{i}$ are semi-stable of degree zero, the bundles $K_{i}$ are also semi-stable of degree zero. We now apply Lemma 3.1 to the line bundle $L_{i}$ of degree $-(\ell-1)(g-1)$ and use the fact that Quot ${ }^{\mathrm{rk}\left(\mathrm{K}_{\mathrm{i}}\right), 0}\left(F_{*}\left(\mathrm{~L}_{\mathrm{i}}\right)\right) \neq \emptyset-$ since this Quot-scheme contains $\left[K_{i}\right]$ - and we obtain that $\operatorname{rk}\left(K_{i}\right) \geq \ell$. Thus we have a homomorphism

$$
\oplus_{i=1}^{q} K_{i} \rightarrow E
$$

which is injective and so both bundles have the same rank and degree. So this map is an isomorphism. Moreover, we observe that $K_{i}=E_{i}$ for any $i=1, \ldots, q$. Thus we have shown that $\Phi$ induces a bijection at the level of $k$-rational points between $\prod_{i=1}^{q}$ Quot $^{\ell, 0}\left(F_{*}\left(\mathrm{~L}_{\mathrm{i}}\right)\right)(k)$ and $\Omega(Q)(k)$.

Since by [JP15, Theorem 6.2.1] each Quot ${ }^{\ell, 0}\left(F_{*}\left(\mathrm{~L}_{\mathrm{i}}\right)\right)(k)$ is a finite set, so $\Omega(Q)(k)$ is also a finite set. Since $k$ is algebraically closed, we deduce that

$$
\operatorname{dim} \Omega(Q)=0
$$

Remark 3.6. If the line bundles $L_{i}$ are not distinct, one still can show finiteness of $\Omega(Q)$ defined as the residual component of the Quot-schemes $\operatorname{Quot}^{\ell \mathrm{q}, 0}\left(F_{*}(\tilde{\mathrm{Q}})\right)$ for any subbundle $\tilde{Q} \subset Q$ of degree 0 and rank $q-1$. 
Remark 3.7. We will define in Section 7 an open subscheme $\Omega(Q) \subset \operatorname{Quot}^{\ell q, 0}\left(F_{*}(Q)\right)$ for any semi-stable vector bundle $Q$, generalizing the above $\Omega(Q)$.

\section{Opers of type $q$}

We now recall the definition of our main object (see [JP15, Definition 3.1.1]).

Definition 4.1. An oper of type $q$ on a smooth projective curve $X$ is a triple $\left(V, \nabla, V_{\bullet}\right)$, where

(1) $V$ is a vector bundle of rank $r=\ell q$ on $X$,

(2) $\nabla: V \rightarrow V \otimes \Omega_{X}^{1}$ is an integrable connection on $V$,

(3) $V_{\bullet}$ is a filtration of length $\ell$ on $V$

$$
0=V_{\ell} \subset V_{\ell-1} \subset \cdots \subset V_{1} \subset V_{0}=V
$$

such that

(a) $V_{0} / V_{1}=Q$ is a vector bundle of rank $q$, and

(b) $\nabla$ induces $\mathcal{O}_{X}$-linear isomorphisms for $1 \leq i \leq \ell-1$

$$
\operatorname{gr}^{i}(V) \rightarrow \operatorname{gr}^{i-1}(V) \otimes \Omega_{X}^{1}
$$

where we define $\operatorname{gr}^{i}(V):=V_{i} / V_{i+1}$ for all $0 \leq i \leq \ell-1$.

Note that our terminology suppresses the dependence on $Q$, but occasionally we may need to emphasize the dependence on $Q$ and in such contexts will refer to such a triple as an oper of type $Q$.

From the definition it is immediate that one has, for all $0 \leq i \leq \ell-1$, isomorphisms of bundles

$$
\operatorname{gr}^{i}(V) \simeq Q \otimes\left(\Omega_{X}^{1}\right)^{\otimes i},
$$

and

$$
\operatorname{det}\left(V_{i}\right)=\operatorname{det}(Q)^{\ell-i} \otimes\left(\Omega_{X}^{1}\right)^{q((\ell-1)+(\ell-2)+\cdots+i)}=\operatorname{det}(Q)^{\ell-i} \otimes\left(\Omega_{X}^{1}\right)^{\frac{q(\ell-i)(\ell+i-1)}{2}}
$$

Note that we have the following equivalence

$$
\operatorname{deg}(Q)=-q(\ell-1)(g-1) \Longleftrightarrow \operatorname{deg}(V)=0 .
$$

In the next proposition we will show that opers of type $q$ exist over an algebraically closed field of any characteristic. We first recall that $\mathscr{G}_{\ell}$ denotes the Gunning bundle of rank $\ell$ associated to the thetacharacteristic $\theta$. We equip $\mathscr{G}_{\ell}$ with any connection $\nabla_{\mathscr{G}_{\ell}}$ and with its natural filtration $\mathscr{G}_{\bullet}$. Then it is well-known that the triple $\left(\mathscr{G}_{\ell}, \nabla_{\mathscr{G}_{\ell}}, \mathscr{G}_{\bullet}\right)$ is an oper (of type 1) under the assumption $p>(\ell-1)(g-1)$, if $\operatorname{char}(k)=p>0$.

Proposition 4.4. Let $k$ be any algebraically closed field. If $\operatorname{char}(k)=p>0$, we assume that $p>q(\ell-1)(g-1)$. Let $S$ be a stable vector bundle on $X$ with $\operatorname{deg}(S)=0$ and $\operatorname{rk}(S)=q$. Then the triple

$$
\left(V=\mathscr{G}_{\ell} \otimes S, \nabla, V_{\bullet}=\mathscr{G}_{\bullet} \otimes S\right)
$$

is an oper of type q for any connection $\nabla$ on $\mathscr{G}_{\ell} \otimes S$.

Proof. The proof is elementary. Let us note that the space of connections on $\mathscr{G}_{\ell} \otimes S$ is non-empty, since by stability the degree- 0 vector bundle $S$ admits a connection $\nabla_{S}$, so $\nabla_{\mathscr{G}_{\ell}} \otimes \nabla_{S}$ is a connection on $\mathscr{G}_{\ell} \otimes S$. Note that, again by stability of $S$, the filtration $V_{\bullet}$ coincides with the Harder-Narasimhan filtration of $V$. The assumption on $p$ implies that no proper subbundle of the filtration $\mathscr{G}_{\bullet} \otimes S$ admits a connection. These observations easily show that any connection $\nabla$ on $V$ is an oper connection. Finally we note that $V_{0} / V_{1}=S \otimes \theta^{-(\ell-1)}$. 
We say that an oper $\left(V, \nabla, V_{\bullet}\right)$ of type $q$ is nilpotent (resp. dormant) if the oper connection $\nabla$ is nilpotent of exponent at most $\mathrm{rk}(V)$ (resp. has $p$-curvature zero). Before proceeding further let us recall the following result of [JP15, Theorem 3.1.6] which shows that dormant opers of type $q$ exist.

Theorem 4.5. Let $Q$ be any vector bundle of rank q. Then the triple

$$
\left(F^{*}\left(F_{*}(Q)\right), \nabla^{\text {can }}, F^{*}\left(F_{*}(Q)\right)_{\bullet}\right)
$$

forms a dormant oper of type q. Here $\nabla^{\text {can }}$ denotes the canonical connection on the vector bundle $F^{*}\left(F_{*}(Q)\right)$.

The relationship between opers of type 1 and the Quot-scheme $\operatorname{Quot}^{r, 0}\left(F_{*}(Q)\right)$ when $Q$ is a line bundle was studied in [JP15]. The main result [JP15, Theorem 6.2.1 and Theorem 5.4.1] is the following

Theorem 4.6. Assume $p>r(r-1)(r-2)(g-1)$. Let $Q$ be a line bundle of degree $\operatorname{deg}(Q)=-(r-1)(g-1)$. Then the set of dormant opers $\left(V, \nabla, V_{\bullet}\right)$ with $V_{0} / V_{1} \simeq Q$ is a non-empty, finite set, in bijection with the set of k-rational points Quot $^{r, 0}\left(F_{*}(Q)\right)(k)$.

We now give an alternative characterization of the underlying bundle $V$ of an oper $\left(V, \nabla, V_{\bullet}\right)$ of type $q$.

Theorem 4.7. Let $X / k$ be a smooth, projective curve over an algebraically closed field $k$. Let $S$ be a vector bundle on $X$. Let $\theta$ be a line bundle on X. Suppose that the following hypotheses are satisfied:

(1) One has $\theta^{2} \simeq \Omega_{X}^{1}$ i.e. $\theta$ is a theta-characteristic on $X$.

(2) $\operatorname{deg}(S)=0$.

(3) an isomorphism $\mathscr{E} n d(S)=\mathcal{O}_{X} \oplus \mathscr{E} n d_{0}(S)$ of $\mathcal{O}_{X}$-modules.

(4) $S$ is stable.

(5) $\mathscr{E} n d_{0}(S)^{*} \simeq \mathscr{E} n d_{0}(S)$.

Then for every integer $\ell \geq 2$ there exists a vector bundle $V$ on $X$ satisfying the following

(1) $\operatorname{deg}(V)=0$

(2) $V$ is equipped with a fltration $V=V_{0} \supset V_{1} \supset V_{2} \supset \cdots \supset V_{\ell}=0$,

(3) $\operatorname{gr}^{i}(V) \simeq S \otimes \theta^{2 i-(\ell-1)}=: S_{i}$

(4) for all $i=0,1, \ldots, \ell-1$ the extension

$$
0 \rightarrow \mathrm{gr}^{i+1}(V) \rightarrow V_{i} / V_{i+2} \rightarrow \mathrm{gr}^{i}(V) \rightarrow 0
$$

is the unique non-split extension of this type.

Up to an isomorphism, there is only one vector bundle $V$ with these properties.

Remark 4.8. If $\operatorname{rk}(Q)$ is not divisible by $p$ then one has an isomorphism $\mathscr{E} n d(S)=\mathcal{O}_{X} \oplus \mathscr{E} n d_{0}(S)$. Further one then also has $\mathscr{E} n d_{0}(S)^{*} \simeq \mathscr{E} n d_{0}(S)$. Hence in particular the hypotheses of the theorem can easily be satisfied for any stable bundle $S$ of degree zero and of rank coprime to the characteristic of the ground field. In particular these hypotheses are easily satisfied in characteristic zero.

We begin with the following lemma.

Lemma 4.9. Let $\theta_{i}=\theta^{2 i-(\ell-1)}$. Then for all $i \geq 1$,

$$
\theta_{i-1}^{*} \otimes \theta_{i} \simeq \Omega_{X}^{1} \text {. }
$$

Proof. From the definition

$$
\theta_{i-1}^{*} \otimes \theta_{i}=\theta^{-(2(i-1)-(\ell-1))+(2 i-(\ell-1))} \simeq \theta^{2 i-2(i-1)} \simeq \theta^{2} \simeq \Omega_{X}^{1} .
$$

Hence the claim.

Lemma 4.10. For $i=0, \ldots, \ell-1$ one has

$$
\operatorname{Ext}^{1}\left(S_{i-1}, S_{i}\right) \simeq H^{1}\left(X, \Omega_{X}^{1}\right) .
$$


Proof. One has

$$
\begin{aligned}
\operatorname{Ext}^{1}\left(S_{i-1}, S_{i}\right) & =H^{1}\left(X, \mathscr{H} \circ \mathrm{om}\left(S_{i-1}, S_{i}\right)\right) \\
& =H^{1}\left(X, S_{i-1}^{*} \otimes S_{i}\right) \\
& =H^{1}\left(X, S^{*} \otimes S \otimes \theta_{i-1}^{*} \otimes \theta_{i}\right) \\
& =H^{1}\left(X, S^{*} \otimes S \otimes \Omega_{X}^{1}\right)[\text { by Lemma 4.9]. }
\end{aligned}
$$

Hence

$$
\begin{aligned}
\operatorname{Ext}^{1}\left(S_{i-1}, S_{i}\right) & \simeq H^{1}\left(X, S^{*} \otimes S \otimes \Omega_{X}^{1}\right) \\
& =H^{1}\left(X,\left(\mathcal{O}_{X} \oplus \mathscr{E} n d_{0}(S)\right) \otimes \Omega_{X}^{1}\right) \\
& =H^{1}\left(X, \Omega_{X}^{1}\right) \oplus H^{1}\left(X, \mathscr{E} n d_{0}(S) \otimes \Omega_{X}^{1}\right) .
\end{aligned}
$$

By Serre duality

$$
H^{1}\left(X, \mathscr{E} n d_{0}(S) \otimes \Omega_{X}^{1}\right) \simeq H^{0}\left(X, \mathscr{E} n d_{0}(S)\right),
$$

and as $S$ is stable of degree zero (by hypothesis) so $H^{0}\left(X, \mathscr{E} n d_{0}(S)\right)=0$. This proves the claim.

Lemma 4.18. For $i=2, \ldots, \ell-1$ one has

$$
\operatorname{Ext}^{1}\left(S_{i-2}, S_{i}\right)=0
$$

Proof. One has

$$
\begin{aligned}
\operatorname{Ext}^{1}\left(S_{i-2}, S_{i}\right) & \simeq H^{1}\left(X, \mathscr{H} \circ \mathrm{om}\left(S_{i-2}, S_{i}\right)\right) \\
& =H^{1}\left(X, S^{*} \otimes S \otimes \theta_{i-2}^{*} \otimes \theta_{i}\right) \\
& =H^{1}\left(X, \theta_{i-2}^{*} \otimes \theta_{i} \oplus \mathscr{E} n d_{0}(S) \otimes \theta_{i-2}^{*} \otimes \theta_{i}\right) .
\end{aligned}
$$

Now

Therefore

$$
\theta_{i-2}^{*} \otimes \theta_{i} \simeq \theta^{-(2(i-2)-(\ell-1))+(2 i-(\ell-1))} \simeq \theta^{-2 i+4+2 i} \simeq \Omega_{X}^{1 \otimes 2}
$$

$$
H^{1}\left(X, \theta_{i-2}^{*} \otimes \theta_{i}\right)=H^{1}\left(X, \Omega_{X}^{1} \otimes 2\right)=0
$$

and

$$
H^{1}\left(X, \mathscr{E} n d_{0}(S) \otimes \Omega_{X}^{1 \otimes 2}\right) \simeq H^{0}\left(X, \mathscr{E} n d_{0}(S) \otimes\left(\Omega_{X}^{1}\right)^{-1}\right)=0
$$

as $S$ is stable, hence there are no global homomorphisms $S \rightarrow S \otimes\left(\Omega_{X}^{1}\right)^{-1}$ since $\mu\left(S \otimes\left(\Omega_{X}^{1}\right)^{-1}\right)<\mu(S)$. This proves the lemma.

Lemma 4.22. For all $j \geq 3$ one has

$$
H^{1}\left(X, \mathscr{E} n d(S) \otimes \theta^{j}\right)=0 .
$$

Proof. This is clear by stability of $S$ and Serre duality.

Lemma 4.23. For $j_{0}>i_{0}+1$ we have

$$
\operatorname{Ext}^{1}\left(S_{i_{0}-1}, S_{j_{0}-1}\right)=0
$$

Proof. We have

$$
\operatorname{Ext}^{1}\left(S_{i_{0}-1}, S_{j_{0}-1}\right) \simeq H^{1}\left(\mathscr{E} n d(S) \otimes \theta^{2\left(j_{0}-i_{0}\right)}\right)
$$

and as $j_{0}>i_{0}+1$ so $j_{0}-i_{0}>1$. Hence this space is of the form $H^{1}\left(\mathscr{E} n d(S) \otimes \theta^{m}\right)$ with $m \geq 3$. So we are done by Lemma 4.22 .

Lemma 4.24. Suppose for some $i_{0} \geq 0$ the bundle $V_{i_{0}}$ has been constructed with asserted properties. Then one has

$$
\operatorname{Ext}^{1}\left(S_{i_{0}-1}, V_{j}\right)=0 \quad \text { for } j \geq i_{0}+1 \text {. }
$$


Proof. Clearly the claim is true for $j=\ell$ as $V_{\ell}=0$. We prove the claim by descending induction on $j$. Suppose the lemma is true for some $j_{0}$ with $i_{0}+1<j_{0} \leq \ell$. Then we claim that the assertion is also true for $j_{0}-1$. As $j_{0}>i_{0}+1$, one has the exact sequence

$$
0 \rightarrow V_{j_{0}} \rightarrow V_{j_{0}-1} \rightarrow S_{j_{0}-1} \rightarrow 0 .
$$

Then applying $\operatorname{Hom}\left(S_{i_{0}-1},-\right)$ one gets

$$
\rightarrow \operatorname{Ext}^{1}\left(S_{i_{0}-1}, V_{j_{0}}\right) \rightarrow \operatorname{Ext}^{1}\left(S_{i_{0}-1}, V_{j_{0}-1}\right) \rightarrow \operatorname{Ext}^{1}\left(S_{i_{0}-1}, S_{j_{0}-1}\right) \rightarrow 0 .
$$

By induction hypothesis the first term is zero and by Lemma 4.23 the last term is zero. Hence the middle term is zero and the claim is proved.

Now we are ready to prove Theorem 4.7.

Proof of Theorem 4.7. The construction of the bundle $V$ whose existence is asserted in the theorem is inductive. We let $V_{\ell}=0$ and $V_{\ell-1}=S_{\ell-1}$. Now we define $V_{\ell-2}$ as the unique non-split extension

$$
0 \rightarrow S_{\ell-1}=V_{\ell-1} \rightarrow V_{\ell-2} \rightarrow S_{\ell-2} \rightarrow 0,
$$

which is given by the isomorphism of Lemma 4.10:

$$
\operatorname{Ext}^{1}\left(S_{\ell-2}, S_{\ell-1}\right) \simeq H^{1}\left(X, \Omega_{X}^{1}\right) .
$$

Now, if $\ell-3 \geq 0$ we define $V_{\ell-3}$ using $V_{\ell-2}$ as follows. Apply $\operatorname{Hom}\left(S_{\ell-3},-\right)$ to the exact sequence defining $V_{\ell-2}$. This gives

$$
\begin{aligned}
0 \rightarrow \operatorname{Hom}\left(S_{\ell-3}, S_{\ell-1}\right) \rightarrow \operatorname{Hom}\left(S_{\ell-3}, V_{\ell-2}\right) & \rightarrow \operatorname{Hom}\left(S_{\ell-3}, S_{\ell-2}\right) \rightarrow \\
& \rightarrow \operatorname{Ext}^{1}\left(S_{\ell-3}, S_{\ell-1}\right) \rightarrow \operatorname{Ext}^{1}\left(S_{\ell-3}, V_{\ell-2}\right) \rightarrow \operatorname{Ext}^{1}\left(S_{\ell-3}, S_{\ell-2}\right) \rightarrow 0 .
\end{aligned}
$$

By Lemma 4.18 we get $\operatorname{Ext}^{1}\left(S_{\ell-3}, S_{\ell-1}\right)=0$, and by Lemma 4.10 we get

$$
\operatorname{Ext}^{1}\left(S_{\ell-3}, S_{\ell-2}\right)=H^{1}\left(X, \Omega_{X}^{1}\right) \text {. }
$$

Therefore we get

$$
\operatorname{Ext}^{1}\left(S_{\ell-3}, V_{\ell-2}\right) \simeq H^{1}\left(X, \Omega_{X}^{1}\right) .
$$

So we define $V_{\ell-3}$ as the unique non-split extension given by this isomorphism. Then we have by construction

$$
0 \rightarrow V_{\ell-2} \rightarrow V_{\ell-3} \rightarrow S_{\ell-3} \rightarrow 0 .
$$

Now we repeat this process to obtain the general construction. Suppose that for some $i_{0} \geq 0, V_{i_{0}}$ has been constructed with the asserted properties. Then one has an exact sequence

$$
0 \rightarrow V_{i_{0}+1} \rightarrow V_{i_{0}} \rightarrow S_{i_{0}} \rightarrow 0 .
$$

Then we claim that there is a unique non-split extension in $\operatorname{Ext}^{1}\left(S_{i_{0}-1}, V_{i_{0}}\right)$ which gives $V_{i_{0}-1}$. We proceed as follows: apply $\operatorname{Hom}\left(S_{i_{0}-1},-\right)$ to the above short exact sequence to get

$$
\rightarrow \operatorname{Ext}^{1}\left(S_{i_{0}-1}, V_{i_{0}+1}\right) \rightarrow \operatorname{Ext}^{1}\left(S_{i_{0}-1}, V_{i_{0}}\right) \rightarrow \operatorname{Ext}^{1}\left(S_{i_{0}-1}, S_{i_{0}}\right) \rightarrow 0 .
$$

By Lemma 4.10 one as $\operatorname{Ext}^{1}\left(S_{i_{0}-1}, S_{i_{0}}\right) \simeq H^{1}\left(X, \Omega_{X}^{1}\right)$, and so to prove our claim it suffices to prove that $\operatorname{Ext}^{1}\left(S_{i_{0}-1}, V_{i_{0}+1}\right)=0$. This follows from Lemma 4.24. Repeating this process eventually one gets $V_{0}$ as the unique non-split extension

$$
0 \rightarrow V_{1} \rightarrow V_{0} \rightarrow Q_{0} \rightarrow 0
$$

This completes the proof. 


\section{Properties of the Quot-scheme $\operatorname{Quot}^{r, 0}\left(F_{*}(Q)\right)$ when $q=1$}

The following proposition gives some properties on the Quot-scheme $\operatorname{Quot}^{r, 0}\left(F_{*}(Q)\right)$ when $Q$ is a line bundle. It generalizes the case $r=2$, which was already shown in [JP15, Lemma 7.1.3] and in the proof of [JP15, Theorem 7.1.2].

We also note that there is a gap in the proof of [JP15, Theorem 7.1.2] and therefore we shall give the proof of the following theorem with all the details.

Let $Q$ be a line bundle of degree $\operatorname{deg}(Q)=-(r-1)(g-1)+d$ with $d \geq 0$ and let $\mathscr{C}$ be an irreducible component of $\operatorname{Quot}^{r, 0}\left(F_{*}(Q)\right)$. We recall from Proposition 2.4(5) that the expected dimension of $\mathscr{C}$ is $r d$.

Definition 5.1. We will say that $\mathscr{C}$ contains a dormant oper if there exists an effective divisor $D$ of degree $d$ and a point $[E] \in \operatorname{Quot}^{r, 0}\left(F_{*}(Q(-D))\right)$ - hence $[E]$ corresponds to a dormant oper — such that $[E] \in \mathscr{C}$ under the natural inclusion

$$
\operatorname{Quot}^{r, 0}\left(F_{*}(Q(-D))\right) \subset \operatorname{Quot}^{r, 0}\left(F_{*}(Q)\right) \text {. }
$$

Remark 5.2. We note that the above definition of $\mathscr{C}$ containing a dormant oper is more restrictive than the natural one: there exists a $[E] \in \mathscr{C}$ such that the triple $\left(F^{*} E, \nabla^{c a n}, F^{*} E_{\bullet}\right)$, where the filtration $F^{*} E_{\bullet}$ is the induced filtration from $F^{*}\left(F_{*}(Q)\right)$, is an oper of type 1 . Note that the latter definition was used in [JP15] for $r=2$. It can be checked that both definitions coincide for small $d$.

Theorem 5.3. With the above notation and assuming that $p>r(r-1)(r-2)(g-1)$ we have for any irreducible component $\mathscr{C}$ containing a dormant oper

(1) $\operatorname{dim}(\mathscr{C})=r d$.

(2) For a general vector bundle $[E] \in \mathscr{C}$ the map $f_{E}$ obtained by adjunction

$$
f_{E}: F^{*}(E) \rightarrow Q
$$

is surjective.

Proof. (1) We prove the result by induction on $d$. For $d=0$ this is exactly [JP15, Theorem 6.2.1]. Assume that the statement holds for an integer $d \geq 0$ and consider a line bundle $Q$ with $\operatorname{deg}(Q)=-(r-1)(g-1)+(d+1)$. Let $\mathscr{C} \subset \operatorname{Quot}^{r, 0}\left(F_{*}(Q)\right)$ be an irreducible component containing a dormant oper [E], i.e. by definition there exists an effective divisor $D$ of degree $d+1$ such that $E \hookrightarrow F_{*}(Q(-D)) \subset F_{*}(Q)$. From now on the proof goes along the lines of [JP15, Lemma 7.1.3]. For the convenience of the reader we include it here.

We decompose $D=x+D^{\prime}$ with $x \in X$ and $D^{\prime}$ effective of degree $d$. Let $\mathscr{C}^{\prime}$ be an irreducible component of $\operatorname{Quot}^{r, 0}\left(F_{*}(Q(-x))\right) \cap \mathscr{C}$ containing $[E]$. By induction we have $\operatorname{dim}\left(\mathscr{C}^{\prime}\right)=r$ d. Now we claim that $\operatorname{codim}_{\mathscr{C}} \mathscr{C}^{\prime} \leq r$. To prove this, note that $\mathscr{C}^{\prime} \neq \emptyset$. Since $\mathscr{C}$ is an irreducible component of the Quot-scheme, it is equipped with a universal quotient sheaf $\mathcal{Q}$ over $X \times \mathscr{C}$.

$$
0 \longrightarrow \mathcal{E} \longrightarrow p_{X}^{*}\left(F_{*}(Q)\right) \longrightarrow \mathcal{Q} \longrightarrow 0
$$

We denote by $\mathcal{E}$ the $\operatorname{kernel} \operatorname{ker}\left(p_{X}^{*}\left(F_{*}(Q)\right) \longrightarrow \mathcal{Q}\right)$. Since $\mathcal{Q}$ and $p_{X}^{*}\left(F_{*}(Q)\right)$ are $\mathscr{C}$-flat, $\mathcal{E}$ is also $\mathscr{C}$-flat and $\forall c \in \mathscr{C}$ the homomorphism $\mathcal{E}_{\mid X \times\{c\}} \rightarrow F_{*}(Q)$ is injective (see e.g. [HL10]). Hence, since $F_{*}(Q)$ is locally free, $\mathcal{E}_{\mid X \times\{c\}}$ is also locally free (since torsion free over a smooth curve) and by [HL10, Lemma 2.1.7] we conclude that $\mathcal{E}$ is locally free over $X \times \mathscr{C}$. Since $p_{X}^{*}\left(F_{*}(Q)\right)=\left(F \times \operatorname{id}_{\mathscr{C}}\right)_{*}\left(p_{X}^{*}(Q)\right)$ we obtain by adjunction a non-zero $\operatorname{map}\left(F \times \operatorname{id}_{\mathscr{C}}\right)^{*}(\mathcal{E}) \rightarrow p_{X}^{*}(Q)$, hence a non-zero global section $\sigma$ of the rank- $r$ vector bundle

$$
\mathcal{V}:=\mathscr{H} \text { om }\left(\left(F \times \operatorname{id}_{\mathscr{C}}\right)^{*}(\mathcal{E}), p_{X}^{*}(Q)\right)
$$

over $X \times \mathscr{C}$. It is clear that $\operatorname{Quot}^{r, 0}\left(F_{*}(Q(-x))\right) \cap \mathscr{C}$ is the zero-scheme of the section $\sigma_{\mid\{x\} \times \mathscr{C}} \in H^{0}\left(\mathscr{C}, \mathcal{V}_{\mid\{x\} \times \mathscr{C}}\right)$ obtained after restriction. Hence $\operatorname{codim}_{\mathscr{C}} \mathscr{C}^{\prime} \leq r$ and therefore $\operatorname{dim}(\mathscr{C}) \leq r d+r$. On the other hand, by 
the dimension estimates of the Quot-schemes in Proposition 2.5(2) we have $\operatorname{dim}(\mathscr{C}) \geq r d+r$. Therefore $\operatorname{dim}(\mathscr{C})=r d+r$ and we are done.

(2) First consider the case $d=0$. Then by [JP15, Theorem 5.4.1 (1)] any $[E] \in \operatorname{Quot}^{r, 0}\left(F_{*}(Q)\right)$ is an oper, which implies that $f_{E}^{*}: F^{*}(E) \rightarrow Q$ is surjective.

We now assume that $d>0$. With the previous notation we denote by $\sum$ the zero-scheme of the global section $\sigma$ of the vector bundle $\mathcal{V}$ and by $p_{X}$ and $p_{\mathscr{C}}$ projections onto $X$ and $\mathscr{C}$ respectively. Thus one has a diagram

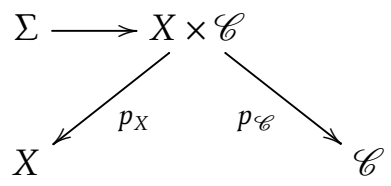

We have the set-theoretical equalities

$$
\begin{aligned}
\sum_{\mid\{x \mid \times \mathscr{C}} & =\operatorname{Quot}^{r, 0}\left(F_{*}(Q(-x))\right) \cap \mathscr{C} \\
& =\left\{E \in \mathscr{C}: f_{E}: F^{*}(E) \rightarrow Q \text { not surjective at } x\right\} .
\end{aligned}
$$

Since $\Sigma$ is closed and $p_{\mathscr{C}}$ is a proper map, so $\Sigma^{\prime}=p_{\mathscr{C}}(\Sigma)$ is a closed subset of the irreducible component $\mathscr{C}$. So there are two possibilities:

(I) Either $\Sigma^{\prime} \neq \mathscr{C}$, or

(II) $\Sigma^{\prime}=\mathscr{C}$.

Now suppose we are in Case (I): in this case for a general $[E] \in \mathscr{C}$, one has $(X \times\{E\}) \cap \sum=\emptyset$, which is equivalent to surjectivity of $f_{E}$. So in Case (I) one has surjectivity of $f_{E}$ for general $[E]$ as claimed.

So let us suppose we are in Case (II). In this case there is at least one irreducible component of $\Sigma$, which we will again denote by $\Sigma$, which surjects onto $\mathscr{C}$ under $p_{\mathscr{C}}$. Hence

$$
\operatorname{dim}(\Sigma) \geq \operatorname{dim}(\mathscr{C}) \geq r d
$$

Consider the restriction $\bar{p}_{X}: \Sigma \rightarrow X$ of $p_{X}: X \times \mathscr{C} \rightarrow X$ to $\Sigma$. First we assume that $\bar{p}_{X}$ is surjective. Since $X$ is smooth and $\Sigma$ is irreducible, by [Har77, Chapter III, Proposition 9.7] $\bar{p}_{X}$ is flat. Hence by [Har77, Chapter III, Corollary 9.6] any irreducible component of the fiber $\bar{p}_{X}^{-1}(x)$ has dimension equal to $\operatorname{dim}(\Sigma)-1 \geq r d-1$. By assumption $\mathscr{C}$ contains a dormant oper $[E]$ and as $\Sigma^{\prime}=\mathscr{C}$, there exists an $x_{0} \in X$ such that $\left(x_{0},[E]\right) \in \Sigma$. Since any irreducible component of $\bar{p}_{X}^{-1}\left(x_{0}\right)$ has dimension $\geq r d-1$, we see that the irreducible component of $\bar{p}_{X}^{-1}\left(x_{0}\right)$ containing [E] has dimension at least $r d-1$ and this irreducible component is contained in $\operatorname{Quot}^{r, 0}\left(F_{*}\left(Q\left(-x_{0}\right)\right)\right)$. But this contradicts part (1) with $d-1 \geq 0$.

Finally, we need to consider the case when $\bar{p}_{X}$ is not surjective. Since $\Sigma$ is irreducible, $\bar{p}_{X}(\Sigma)=\left\{x_{0}\right\}$ for some point $x_{0} \in X$. Hence $\Sigma \subset \bar{p}_{X}^{-1}\left(x_{0}\right)=\left\{x_{0}\right\} \times \mathscr{C}$, which implies that

$$
\Sigma \subset \operatorname{Quot}^{r, 0}\left(F_{*}\left(Q\left(-x_{0}\right)\right)\right) \cap \mathscr{C} \text {. }
$$

By assumption $\mathscr{C}$ contains a dormant oper $[E]$, hence there exists an effective divisor $D$ of degree $d \geq 1$ such that $[E] \in \operatorname{Quot}^{r, 0}\left(F_{*}(Q(-D))\right)$. Then clearly $x_{0} \in D$ and $\left(x_{0},[E]\right) \in \Sigma$. We then obtain a contradiction, since $\operatorname{dim}(\Sigma) \geq r d$ and by part (1) the irreducible component of Quot ${ }^{r, 0}\left(F_{*}\left(Q\left(-x_{0}\right)\right)\right)$ containing $[E]$ has dimension $r(d-1)<r d$.

Remark 5.5. The gap in the proof of [JP15, Theorem 7.1.2] is related to the fact that the intersection $\operatorname{Quot}^{2,0}\left(F_{*}(Q(-x))\right) \cap \mathscr{C}$ is not necessarily irreducible. 


\section{The Quot-scheme $\operatorname{Quot}^{r, 0}\left(F_{*}(Q)\right)$ is bigger than expected when $q \geq 2$}

We assume that $q=\operatorname{rk}(Q) \geq 2$ and that

$$
-(r-q)(g-1) \leq \operatorname{deg}(Q)=-(r-q)(g-1)+d \leq-1,
$$

or equivalently,

$$
0 \leq d \leq(r-q)(g-1)-1 .
$$

Note that the last inequality implies that $d<(r-1)(g-1)-1$. With this notation we have the following result.

Proposition 6.1. Let $[E] \in \operatorname{Quot}^{\mathrm{r}, 0}\left(F_{*}(\mathrm{Q})\right)$. Then we have

$$
\operatorname{dim} \operatorname{Quot}^{\mathrm{r}, 0}\left(F_{*}(\mathrm{Q})\right)>\exp \cdot \operatorname{dim}_{[E]} \operatorname{Quot}^{\mathrm{r}, 0}\left(F_{*}(\mathrm{Q})\right)=r d \text {. }
$$

Proof. By [JP15, Theorem 2.3.1] there exists a line subbundle $L \subset Q$ such that

$$
\begin{aligned}
\operatorname{deg} L & \geq \mu(Q)-\left(1-\frac{1}{q}\right)(g-1)-\left(1-\frac{1}{q}\right) \\
& =\left(-\frac{r}{q}+1\right)(g-1)+\frac{d}{q}-\left(1-\frac{1}{q}\right)(g-1)-\left(1-\frac{1}{q}\right) \\
& =-\frac{1}{q}(r-1)(g-1)+\frac{d}{q}-\left(1-\frac{1}{q}\right) .
\end{aligned}
$$

Note that the expression on the right-hand side is not necessarily an integer. We then introduce the quantity $\delta=\operatorname{deg} L+(r-1)(g-1)$. Then clearly

$$
\operatorname{Quot}^{\mathrm{r}, 0}\left(F_{*}(\mathrm{~L})\right) \subset \operatorname{Quot}^{\mathrm{r}, 0}\left(F_{*}(\mathrm{Q})\right)
$$

and by Proposition $2.5(2) \operatorname{dim} \operatorname{Quot}^{\mathrm{r}, 0}\left(F_{*}(\mathrm{~L})\right) \geq r \delta$. Therefore in order to show the proposition it will be enough to show the inequality $\delta>d$. We have

$$
\begin{aligned}
\delta & =\operatorname{deg} L+(r-1)(g-1), \\
& \geq(r-1)(g-1)\left(1-\frac{1}{q}\right)+\frac{d}{q}-\left(1-\frac{1}{q}\right), \\
& =\left(1-\frac{1}{q}\right)[(r-1)(g-1)-1]+\frac{d}{q} .
\end{aligned}
$$

Now we observe that the inequality

$$
\left(1-\frac{1}{q}\right)[(r-1)(g-1)-1]+\frac{d}{q}>d
$$

is equivalent to

$$
(r-1)(g-1)-1>d,
$$

which holds by assumption on $d$.

Remark 6.8. In particular if $e(E)=0$ the Quot-scheme Quot ${ }^{\mathrm{r}, 0}\left(F_{*}(\mathrm{Q})\right)$ has some positive-dimensional components, as already observed in the previous section, see also Theorem 3.5. 


\section{A characterization of dormant opers of type $q$}

Lemma 7.1. Let $Q$ be any vector bundle of rank $q$ and $\operatorname{deg}(Q)=-q(\ell-1)(g-1)$. We denote by $\left(V, \nabla, V_{\bullet}\right)$ the oper $\left(F^{*}\left(F_{*}(Q)\right), \nabla^{c a n}, F^{*}\left(F_{*}(Q)\right)_{\bullet}\right)$ introduced in Theorem 4.5. Let $\left[W^{\prime}\right] \in \operatorname{Quot}^{r, 0}\left(F_{*}(Q)\right)$ and let $W=F^{*}\left(W^{\prime}\right) \subset V$. Then the triple $\left(W, \nabla^{\text {can }}, W_{\bullet}\right)$, where $\nabla^{\text {can }}$ is the canonical connection on $F^{*}\left(W^{\prime}\right)$ and $W_{\bullet}$ the induced flag defined by $W_{i}=W \cap V_{i}$, is an oper of type $q$ if and only if

$$
W_{\ell}=W \cap V_{\ell}=\{0\} .
$$

Proof. Suppose that the triple $\left(W, \nabla^{c a n}, W_{\bullet}\right)$ is an oper of type $q$. By [JP15, Lemma 3.4.2(i)] we have an inclusion $W_{0} / W_{1} \subset Q$ and since both vector bundles have same degree and rank, they are isomorphic. It also follows from [JP15, Lemma 3.4.2(ii)] that $\operatorname{rk}\left(W_{\ell}\right)=0$, hence $W_{\ell}=\{0\}$.

Conversely, assume that $W_{\ell}=\{0\}$. Then consider the sequence $r_{i}=\operatorname{rk}\left(W_{i} / W_{i+1}\right)$. So by [JP15, Lemma 3.4.2(ii)] we have the inequalities

$$
q \geq r_{0} \geq r_{1} \geq \cdots \geq r_{\ell}=0 .
$$

As $\sum_{i=0}^{\ell-1} r_{i}=q \ell$ it follows that all $r_{i}=q$. Thus $\operatorname{rk}\left(W_{i} / W_{i+1}\right)=r_{i}=q$ for all $i$.

Now consider the increasing sequence of degrees $\operatorname{deg}\left(V_{i} / V_{i+1}\right)=\operatorname{deg}(Q)+i q(2 g-2)$ for $i=0, \ldots, \ell-1$. Since we have an injective map $W_{i} / W_{i+1} \hookrightarrow V_{i} / V_{i+1}$ and both the bundles are of the same rank, we obtain $\operatorname{deg}\left(W_{i} / W_{i+1}\right) \leq \operatorname{deg}\left(V_{i} / V_{i+1}\right)$ and one has

$$
0=\sum_{i=0}^{\ell-1} \operatorname{deg}\left(W_{i} / W_{i+1}\right) \leq \sum_{i=0}^{\ell-1} \operatorname{deg}\left(V_{i} / V_{i+1}\right) .
$$

This gives

$$
0=\sum_{i=0}^{\ell-1} \operatorname{deg}\left(W_{i} / W_{i+1}\right) \leq \sum_{i=0}^{\ell-1}(\operatorname{deg}(Q)+i q(2 g-2)) .
$$

The last sum evaluates to

$$
0=\sum_{i=0}^{\ell-1} \operatorname{deg}\left(W_{i} / W_{i+1}\right) \leq q(g-1)(\ell(\ell-1)-\ell(\ell-1))=0 .
$$

Thus one sees that $\operatorname{deg}\left(W_{i} / W_{i+1}\right)=\operatorname{deg}\left(V_{i} / V_{i+1}\right)$. Therefore the above injective maps are isomorphisms: $W_{i} / W_{i+1} \simeq V_{i} / V_{i+1}$ for $i=0, \ldots, \ell-1$. This proves the assertion.

One important consequence of this lemma is the following fundamental result.

Theorem 7.2. Let $Q$ be a semi-stable vector bundle with $\operatorname{deg}(Q)=-q(\ell-1)(g-1)$ and $\operatorname{rk}(Q)=q$ for some integer $\ell \geq 2$. We put $r=\ell q$ and use the notation of Lemma 7.1.

(1) For every $\left[W^{\prime}\right] \in \operatorname{Quot}^{r, 0}\left(F_{*}(Q)\right)$ satisfying

$$
W_{\ell}=W \cap V_{\ell}=\{0\}
$$

the triple $\left(W=F^{*}\left(W^{\prime}\right), \nabla^{c a n}, W_{\bullet}\right)$ is a dormant oper of type $q$.

(2) If $p>r(r-1)(g-1)$ then conversely every dormant oper $\left(W, \nabla, W_{\bullet}\right)$ of type $q$ with $W_{0} / W_{1} \simeq Q$ is of the form $\left(W=F^{*}\left(W^{\prime}\right), \nabla^{\text {can }}, W_{\bullet}\right)$ for some $W^{\prime} \in \operatorname{Quot}^{r, 0}\left(F_{*}(Q)\right)$.

Proof. The first assertion is immediate from Lemma 7.1.

Now suppose $\left(W, \nabla, W_{\bullet}\right)$ is a dormant oper of type $q$. Then by Cartier's Theorem (see [Kat70]) $W=F^{*}\left(W^{\prime}\right)$ for some vector bundle $W^{\prime}$ and as $W=F^{*}\left(W^{\prime}\right) \rightarrow W_{0} / W_{1}=Q$ one gets by adjunction a non-zero map $W^{\prime} \rightarrow F_{*}(Q)$. Let us first show that $W^{\prime}$ is semi-stable (of degree zero). Suppose this is not the case. Then there exists a subbundle $W^{\prime \prime} \hookrightarrow W^{\prime}$ for which $\mu\left(W^{\prime \prime}\right)>0$. Then in fact one has $\mu\left(W^{\prime \prime}\right) \geq \frac{1}{r-1}$. But then 
$F^{*}\left(W^{\prime \prime}\right) \hookrightarrow W$ such that $\mu\left(F^{*}\left(W^{\prime \prime}\right)\right)=p \mu\left(W^{\prime \prime}\right) \geq \frac{p}{r-1}$. On the other hand as $W$ carries the structure of an oper of type $q$, and one has $\mu\left(W_{\ell-1}\right)=q(2(\ell-1)-(\ell-1))(g-1)=q(\ell-1)(g-1)$ and this is the destabilizing subbundle of largest degree, so $\frac{p}{r-1} \leq \mu\left(F^{*}\left(W^{\prime \prime}\right)\right) \leq q(\ell-1)(g-1)$. Hence

$$
p<(r-1) q(\ell-1)(g-1)=(r-1)(r-q)(g-1) .
$$

On the other hand we have assumed that $p>r(r-1)(g-1)$. Therefore we have arrived at a contradiction. Thus the vector bundle $W^{\prime}$ is semi-stable.

Now if $W^{\prime}$ does not map injectively into $F_{*}(Q)$, then the image is a subsheaf of some degree $d \geq 1$ and rank $\leq r-1$ and hence it has slope $\geq \frac{1}{r-1}$. Again by [JP15, Proposition 4.2.1] one sees that $F_{*}(Q)$ does not have any subsheaves of suitably positive degree and of rank $\leq r-1$. So $W^{\prime} \hookrightarrow F_{*}(Q)$.

Proposition 7.3. Using the above notation, the set of points $\left[W^{\prime}\right] \in \operatorname{Quot}^{r, 0}\left(F_{*}(Q)\right)$ satisfying $W_{\ell}=\{0\}$ is an open subset.

Proof. The Quot-scheme Quot ${ }^{r, 0}\left(F_{*}(Q)\right)$ comes equipped with a universal quotient $\mathscr{G}$ defined over the product $X \times \operatorname{Quot}^{r, 0}\left(F_{*}(Q)\right)$ which is flat over $\operatorname{Quot}^{r, 0}\left(F_{*}(Q)\right)$ :

$$
0 \rightarrow \mathscr{W}^{\prime} \rightarrow p_{X}^{*}\left(F_{*}(Q)\right) \rightarrow \mathscr{G} \rightarrow 0 .
$$

Since $p_{X}^{*}\left(F_{*}(Q)\right)$ is locally free, it is flat over Quot ${ }^{r, 0}\left(F_{*}(Q)\right)$ and therefore for any point $y \in \operatorname{Quot}^{r, 0}\left(F_{*}(Q)\right)$ the homomorphism $\mathscr{W}^{\prime} \mid X \times\{y\} \rightarrow F_{*}(Q)$ is injective. Thus $\mathscr{W}^{\prime} \mid X \times\{y\}$ is locally free for any $y$, which in turn implies that $\mathscr{W}^{\prime}$ is locally free. Hence, with the notation of Lemma 7.1 we obtain a homomorphism between locally free sheaves over $X \times \operatorname{Quot}^{r, 0}\left(F_{*}(Q)\right)$

$$
\Phi: F^{*}\left(\mathscr{W}^{\prime}\right) \rightarrow p_{X}^{*}\left(V / V_{\ell}\right)
$$

By Lemma 7.1 the condition $W_{\ell}=\{0\}$, with $W=F^{*}\left(\mathscr{W}^{\prime} \mid X \times\{y\}\right)$, is equivalent to $\Phi_{X \times\{y\}}$ being an isomorphism. But the set of points $y \in \operatorname{Quot}^{r, 0}\left(F_{*}(Q)\right)$ where $\Phi_{X \times\{y\}}$ is not an isomorphism is clearly a closed subset.

We shall denote this open subscheme

$$
\Omega(Q) \subset \operatorname{Quot}^{r, 0}\left(F_{*}(Q)\right) .
$$

We recall that by Theorem 7.2 the open subscheme $\Omega(Q)$ parameterizes dormant opers $\left(W, \nabla, W_{\bullet}\right)$ of type $q$ with $W_{0} / W_{1} \simeq Q$.

Remark 7.5. We observe that the Quot-scheme $\operatorname{Quot}^{r, 0}\left(F_{*}(Q)\right)$ has expected dimension 0 if $\operatorname{rk}(Q)=q$, $\operatorname{deg}(Q)=-q(\ell-1)(g-1)$ and $r=q \ell$, but in the case $q \geq 2$ we have shown in Proposition 6.1 that $\operatorname{Quot}^{r, 0}\left(F_{*}(Q)\right)$ always contains a Quot-scheme Quot ${ }^{r, 0}\left(F_{*}(L)\right)$ for some line subbundle $L \subset Q$, which has dimension $>0$. Clearly, the Quot-scheme Quot ${ }^{r, 0}\left(F_{*}(L)\right)$ is not contained in the open subscheme $\Omega(Q)$ and does not correspond to dormant opers.

Conjecture 7.6. Let $Q$ be a semi-stable vector bundle with $\operatorname{deg}(Q)=-q(\ell-1)(g-1)$ and $\operatorname{rk}(Q)=q$ for some integer $\ell \geq 2$. Put $r=q \ell$. Then, for $p>r(r-1)(r-2)(g-1)$

$\Omega(Q)$ is non-empty and of dimension 0.

We now list some evidence for this conjecture:

(1) If $q=1$, the conjecture is true. This is shown in [JP15] - see Theorem 4.6. Note that in this case we have equality $\Omega(Q)=\operatorname{Quot}^{r, 0}\left(F_{*}(Q)\right)$.

(2) The conjecture is true for a decomposable bundle of the form $Q=\oplus_{i=1}^{q} L_{i}$, where the $L_{i}$ are $q$ distinct line bundles of degree $\operatorname{deg}\left(L_{i}\right)=-(\ell-1)(g-1)$. This is shown in Theorem 3.5. We note that in this case points of $\Omega(Q)$ correspond to direct sums of $q$ opers of type 1 . 


\section{Dimension of Frobenius instability loci}

Throughout this section we assume that $p>r(r-1)(r-2)(g-1)$.

Let $\mathcal{M}(r)$ be the coarse moduli space of semi-stable vector bundles of rank $r$ and degree 0 over $X$. Let $\mathcal{J}(r) \subset \mathcal{M}(r)$ be the closed subscheme of $\mathcal{M}(r)$ parameterizing semi-stable vector bundles $E$ such that $F^{*}(E)$ is not semi-stable. The closed subscheme $\mathcal{J}(r)$ will be referred to as the Frobenius instability locus. The purpose of this section is to make a conjecture on the dimension of $\mathcal{J}(r)$. Before stating the conjecture we recall some facts on the structure of $\mathcal{J}(r)$.

Let $\mathcal{M}(q,-1)$ be the moduli space of semi-stable bundles of rank $q$ and degree -1 over $X$. Note that as we are in the coprime case any semi-stable bundle of rank $q$ and degree -1 is also stable. Let $\mathcal{U}$ be the universal bundle over $X \times \mathcal{M}(q,-1)$. Consider the relative Quot-scheme

$$
\pi: \operatorname{Quot}^{r, 0}\left(\left(F \times \operatorname{id}_{\mathcal{M}(q,-1)}\right)_{*} \mathcal{U}\right) \longrightarrow \mathcal{M}(q,-1)
$$

with fiber $\pi^{-1}(Q)=\operatorname{Quot}^{r, 0}\left(F_{*}(Q)\right)$ over a stable bundle $Q \in \mathcal{M}(q,-1)$.

For $q=1, \ldots, r-1$ we denote by $\mathcal{J}_{q} \subset \mathcal{J}(r)$ the closure of the forgetful map

$$
\alpha_{q}: \operatorname{Quot}^{r, 0}\left(\left(F \times \operatorname{id}_{\mathcal{M}(q,-1)}\right)_{*} \mathcal{U}\right) \longrightarrow \mathcal{M}(r), \quad\left[E \subset F_{*}(Q)\right] \rightarrow E .
$$

Then by [JP15, Theorem 4.4.1] we have the inclusions

$$
\mathcal{J}^{s}(r) \subset \bigcup_{q=1}^{r-1} \mathcal{J}_{q} \subset \mathcal{J}(r),
$$

where $\mathcal{J}^{s}(r)$ denotes the subset of stable vector bundles $E$ such that $F^{*}(E)$ is not semi-stable.

In order to compute the dimension of $\mathcal{J}_{q}$ we need to know the following dimensions

(1) $\operatorname{dim} \operatorname{Quot}^{r, 0}\left(F_{*}(Q)\right)$ for general $Q \in \mathcal{M}(q,-1)$.

(2) $\operatorname{dim} \alpha_{q}^{-1}(E)$ for general $E \in \mathcal{J}_{q}$.

Unfortunately we only know $\operatorname{dim} \mathscr{C}$ for certain irreducible components $\mathscr{C} \subset \operatorname{Quot}^{r, 0}\left(F_{*}(Q)\right)$ when $q=1$.

We therefore focus on the case $q=1$. Let $Q$ be a line bundle of degree -1 and let $\mathscr{C}$ be an irreducible component of $\operatorname{Quot}^{r, 0}\left(F_{*}(Q)\right)$ containing a dormant oper. Then by Theorem $5.3 \operatorname{dim} \mathscr{C}=r((r-1)(g-1)-1)$ and for a general vector bundle $[E] \in \mathscr{C}$ the map $f_{E}: F^{*}(E) \rightarrow Q$ obtained by adjunction is surjective. Let us denote the kernel of $f_{E}$ by $S=\operatorname{ker} f_{E}$. Note that $\operatorname{deg}(S)=1$ for general $[E]$.

With this notation we make the following

Conjecture 8.1. For a general vector bundle $E \in \mathscr{C}$ the bundle $S$ is semi-stable.

As a consequence of this conjecture we obtain that for a general $E \in \mathscr{C}$ the filtration $0 \subset S \subset F^{*}(E)$ is the Harder-Narasimhan filtration of $F^{*}(E)$. Therefore the quotient $F^{*}(E) \rightarrow Q=F^{*}(E) / S$ is uniquely determined and $\operatorname{dim} \operatorname{Hom}\left(E, F_{*}(Q)\right)=1$. This means that the forgetful map $\alpha_{1}$ is generically injective on components $\mathscr{C}$ containing dormant opers.

Hence if Conjecture 8.1 holds, then we have the following formula

$$
\operatorname{dim} \mathcal{J}(r) \geq \operatorname{dim} \mathcal{J}_{1} \geq \operatorname{dim} \mathcal{M}(q,-1)+\operatorname{dim} \mathscr{C}=\left(r^{2}-r+1\right)(g-1)-(r-1) .
$$

Remark 8.2. Conjecture 8.1 holds trivially for $r=2$ and the lower bound of the dimension of $\mathcal{J}(2)$ was already worked out in [JP15, Theorem 7.1.2]. 
Finally, we conjecture the following structure of $\mathcal{J}_{q}$ for any $q=1, \ldots, r-1$.

Conjecture 8.3. The Harder-Narasimhan filtration of $F^{*}(E)$ of a general bundle $E \in \mathcal{J}_{q}$ is minimal, i.e., of the form

$$
0 \subset S \subset F^{*}(E)
$$

where $\operatorname{deg}(S)=1$ and $\operatorname{rk}(S)=r-q$.

Remark 8.4. The above two conjectures hold in the case $p=2, r=2$ by [JRXY06] and for $g=2, r=3, p=3$. This is worked out in [Li19].

\section{References}

[BD00] A. Beilinson and V. Drinfel'd, Quantization of Hitchin's integrable system and Hecke eigensheaves, http://people.math.harvard.edu/_gaitsgde/grad_2009/

[Har77] R. Hartshorne, Algebraic geometry, Graduate Texts in Mathematics, no. 52, Springer-Verlag, New York-Heidelberg, 1977.

[HL10] D. Huybrechts and M. Lehn, The geometry of moduli spaces of sheaves. Second edition. Cambridge Mathematical Library, Cambridge University Press (2010).

[JP15] K. Joshi and C. Pauly, Hitchin-Mochizuki morphism, opers and Frobenius-destabilized vector bundles over curves, Adv. in Math. 274 (2015), 39-75.

[JRXY06] K. Joshi, S. Ramanan, E. Z. Xia and J.-K. Yu, On vector bundles destabilized by Frobenius pull-back, Compos. Math. 142 (2006), no. 3, 616-630.

[JX00] K. Joshi and E. Z. Xia, Moduli of vector bundles on curves in positive characteristics, Compositio Math. 122 (2000), no. 3, 315-321.

[Kat70] N. Katz, Nilpotent connections and the monodromy theorem: Applications of a result of Turrittin, Inst. Hautes Études Sci. Publ. Math. 39 (1970), 175-232.

[Li19] L. Li, Frobenius stratification of moduli spaces of rank 3 vector bundles in characteristic 3, I, Trans. Amer. Math. Soc. 372 (2019), no. 8, 5693-5711.

[Sha76] S. S. Shatz, Degeneration and specialization in algebraic families of vector bundles, Bull. Amer. Math. Soc. 82 (1976), no. 4, 560-562. 